\title{
Serum Polypeptide Alpha-Fetoprotein (AFP) as a possible powerful geroprotector
}

\author{
Alexander Khalyavkin \\ Institute of Biochemical Physics of RAS \\ Federal Research Center"Computer \\ Science and Control" of RAS \\ Moscow, Russia \\ antisenesc@mail.ru
}

\author{
Vyacheslav Krut'ko \\ Federal Research Center "Computer \\ Science and Control" of RAS \\ Sechenov First Moscow State Medical \\ University \\ Moscow, Russia \\ krutkovn@mail.ru
}

\author{
Vitaly Dontsov \\ Federal Research Center "Computer \\ Science and Control" of RAS \\ Moscow, Russia \\ dontsovvi@mail.ru
}

\begin{abstract}
AFP is primarily known as an oncomarker for tumors of the liver and some other organs. But then it was identified immunochemically as a normal fetal serum antigen. Now it is one of the best-known embryo specific proteins with pronounced immunotropic and detoxifying effects and has a very small background serum level in adults. Liver reparative regeneration is accompanied by temporal and malignant growth by constitutive AFP synthesis in adult liver. It is also known that rat sexual activity increased $30 \mathrm{~min}$ after administration of 1 $\mu \mathrm{g} / \mathrm{kg}$ AFP. AFP increased cerebral flow and had pronounced antihypoxic activity. For this reason we studied bioactivating effects of AFP in old mice (females Balb/c aged 18 months) and their survivability. During the two weeks of the experiment, the natural death of animals in the control group was $47 \%$, while AFP administration reduced the mortality up to $17 \%$ in the experimental group. The physical activity of the animals, their habitus and immune status were also higher than in the control group.
\end{abstract}

Keywords - alpha-fetoprotein, geroprotector, survivability, reducing mortality, anti-aging effects

\section{Introduction}

AFP is primarily known as an oncomarker for tumors of the liver and some other organs. But then it was identified immunochemically as a normal fetal serum antigen. Now it is one of the best-known embryo specific proteins with pronounced immunotropic and detoxifying effects and has a very small background serum level in adults. Liver reparative regeneration is accompanied by temporal and malignant growth by constitutive AFP synthesis in adult liver.

It is also known that rat sexual activity increased $30 \mathrm{~min}$ after administration of $1 \mu \mathrm{g} / \mathrm{kg}$ AFP. AFP increased cerebral flow and had pronounced antihypoxic activity. For this reason we studied bioactivating effects of AFP in old mice, keeping in mind their immunoregulatory features, too.

\section{Materials and Methods}

AFP (produced by "Institute of New Medical Technologies", Perm, Russia) was administered to 12 experimental animals (females Balb/c aged 18 months, obtained from kennel Stolbovaya) for 2 weeks, in the morning, intraperitoneally, in $0.5 \mathrm{ml}$ saline, $10 \mu \mathrm{g}$ per $\mathrm{kg}$ of body weight. In 15 control animals, the same saline volume was administered without the drug. In the course of the experiments, we evaluated some parameters of aging as well as survival of animals in the control and experimental groups.
We evaluated the appearance of animals based on the state of their hair - its color and gloss, baldness, and the severity of age-hump. The physical state of the animals was evaluated by the time during which the mice could stay on a string; we took into account the weight of the animals and the relative mass of internal organs ( $\mathrm{mg}$ of organ weight per gram of animal body weight). After the end of the experiments, the remaining animals were sacrificed and a number of parameters important for assessing the level of aging of the animals were measured. To assess immune status, the following parameters were taken into account: relative weight of the immune organs (spleen and thymus) and degree of age related autoaggression based on the level of autoimmune complexes after the addition of polyethylene glycol (PEG6000) to blood serum expressed in units of optical density under spectrophotometric study. The content of intracellular water in tissues was evaluated based on the extent of weight loss (for kidneys) in hypertonic solution ( $40 \%$ sucrose) after $4 \mathrm{~h}$ of incubation.

We traced animal survivability during the studied period (percentage of the original number) and evaluated the mean values of the test parameters and standard deviations for control and experimental values; differences were compared by Student's test, which characterizes the normal distribution of data, and the Mann-Whitney $U$ criterion, which characterizes nonparametric distribution. In the experiments, we used a group of mice at late stages of aging, which was reflected in the mortality level in the population: half of the mice in the vivarium had died during the previous 2 months.

\section{Results and Conclusions}

During the experiment, the natural death of animals in the control group was $47 \%$, while AFP administration reduced the mortality to $17 \%$ in the experimental group. We studied the general state of the surviving animals ( 8 in the control and 10 in the experiment), then the animals were sacrificed, and a number of parameters were examined.

A clear trend towards improvement of the appearance was observed in all the animals treated with AFP: hair loss and bald areas were reduced, hair glistened, and it lost yellowness and patchiness characteristic of old age. Habitus evaluated according to these characteristics was clearly better for all the animals of the experimental group. The physical activity of the animals and their immune status were also higher than in the control group.

Thus, AFP administration drastically increased the survival rate of the oldest mice and had a pronounced bioactivating effect in our experiments. 